\title{
Statistical equilibrium of silicon in the solar atmosphere ${ }^{\star}$
}

\author{
J. R. Shi ${ }^{1,2}$, T. Gehren ${ }^{2}$, K. Butler ${ }^{2}$, L. I. Mashonkina ${ }^{2,3}$, and G. Zhao ${ }^{1,2}$ \\ ${ }^{1}$ National Astronomical Observatories, Chinese Academy of Sciences, Beijing 100012, PR China \\ e-mail: sjr@bao.ac.cn \\ 2 Institut für Astronomie und Astrophysik der Universität München, Scheinerstr. 1, 81679 München, Germany \\ 3 Institute of Astronomy, Russian Academy of Sciences, Pyatnitskaya Str. 48, Moscow, Russia 109017, Russia
}

Received 25 January 2008 / Accepted 23 April 2008

ABSTRACT

\begin{abstract}
Aims. The statistical equilibrium of neutral and ionised silicon in the solar photosphere is investigated. Line formation is discussed and the solar silicon abundance determined.

Methods. High-resolution solar spectra were used to determine solar $\log g f \varepsilon_{\mathrm{Si}}$ values by comparison with Si line synthesis based on LTE and NLTE level populations. The results will be used in a forthcoming paper for differential abundance analyses of metalpoor stars. A detailed analysis of silicon line spectra leads to setting up realistic model atoms, which are exposed to interactions in plane-parallel solar atmospheric models. The resulting departure coefficients are entered into a line-by-line analysis of the visible and near-infrared solar silicon spectrum.

Results. The statistical equilibrium of Si I turns out to depend marginally on bound-free interaction processes, both radiative and collisional. Bound-bound interaction processes do not play a significant role either, except for hydrogen collisions, which have to be chosen adequately for fitting the cores of the near-infrared lines. Except for some near-infrared lines, the NLTE influence on the abundances is weak.

Conclusions. Taking the deviations from LTE in silicon into account, it is possible to calculate the ionisation equilibrium from neutral and ionised lines. The solar abundance based on the experimental $f$-values of Garz corrected for the Becker et al.'s measurement is $7.52 \pm 0.05$. Combined with an extended line sample with selected NIST $f$-values, the solar abundance is $7.52 \pm 0.06$, with a nearly perfect ionisation equilibrium of $\Delta \log \varepsilon_{\odot}(\mathrm{Si}$ II $/ \mathrm{Si}$ I $)=-0.01$.
\end{abstract}

Key words. line: formation - line: profiles - stars: abundances - stars: late-type - Sun: abundances

\section{Introduction}

Neutral silicon gives rise to only a very few and predominantly weak spectral lines in the visible. For that reason it is sometimes regarded as unspectacular and consequently ignored in many abundance analyses. There are at least three important facts that put Si back into perspective:

- silicon is commonly attributed to the $\alpha$ elements that are assumed to be produced in massive type II supernovae. In this case the Galactic chemical evolution of Si should differ significantly from that of iron resulting in logarithmic overabundance ratios $[\mathrm{Si} / \mathrm{Fe}]>0$;

- silicon belongs to the most abundant metals. It competes with $\mathrm{Mg}$ and $\mathrm{Fe}$, which also have similar ionisation energies. It is therefore important as an electron donor in relatively cool turnoff stellar atmospheres, where elements such as $\mathrm{H}$, $\mathrm{C}, \mathrm{N}, \mathrm{O}$ and $\mathrm{Ne}$ with even higher abundances cannot contribute;

- due to its condensation behaviour, $\mathrm{Si}$ is found in many interstellar and interplanetary dust particles and meteorites. Therefore, $\mathrm{Si}$ is the reference element for abundance determinations from solar system meteorites. This is different from the stellar abundance reference, hydrogen.

All three points cannot hide the problem every astronomer encounters when trying to determine the $\mathrm{Si}$ abundance in cool

* Table 1 is only available in electronic form at http://www . aanda.org turnoff stars. Even the line spectrum observed in metal-rich stars is poor, which may be part of the reason why the laboratory work is also far from complete, and some terms and line transitions have not yet been identified unambiguously. On the other hand, spectra of hotter stars such as those of types B or O display a multitude of spectral lines of up to three consecutive ionisation stages, Si II, Si III, and Si IV.

Neutral silicon has only a single strong line in the blue part of the spectrum, i.e. the $3 \mathrm{p}^{1} \mathrm{~S}_{0}-4 \mathrm{~s}{ }^{1} \mathrm{P}_{1}^{\mathrm{o}} 3905 \AA$ line. That line appears in a spectral range that is not easy to observe because

- cool stars have their maximum flux in the red;

- spectrograph coatings are optimized for the visual, and

- CCD detectors have a lower QDE in the blue.

Additionally, the line is blended by a number of other metal lines, and line synthesis requires a careful representation of the blend components, in particular in metal-rich stellar spectra. A second important line is the intercombination line, $3 \mathrm{p}{ }^{1} \mathrm{~S}_{0}-4 \mathrm{~s}^{3} \mathrm{P}_{1}^{\mathrm{o}}$ at $4103 \AA$. Different from other allowed transitions in the visible and the near red, this line is relatively strong (comparable to the $\mathrm{Mg}$ I line at $4571 \AA$ ), and at typical $\mathrm{S} / \mathrm{N}$ of stellar spectra the detection becomes difficult at metallicities $[\mathrm{Fe} / \mathrm{H}]<-2.5$.

The situation becomes increasingly worse in metal-poor stars, even more so if the stars are at the hot end of the turnoff with effective temperatures around 6200 to 6500 K. Here, Si I as a typical minority ion becomes nearly fully ionised leaving practically no neutral silicon atoms in any of the excited levels. Since the triplet lines arising from the $3 \mathrm{p}^{3} \mathrm{P}$ ground state are all in the 


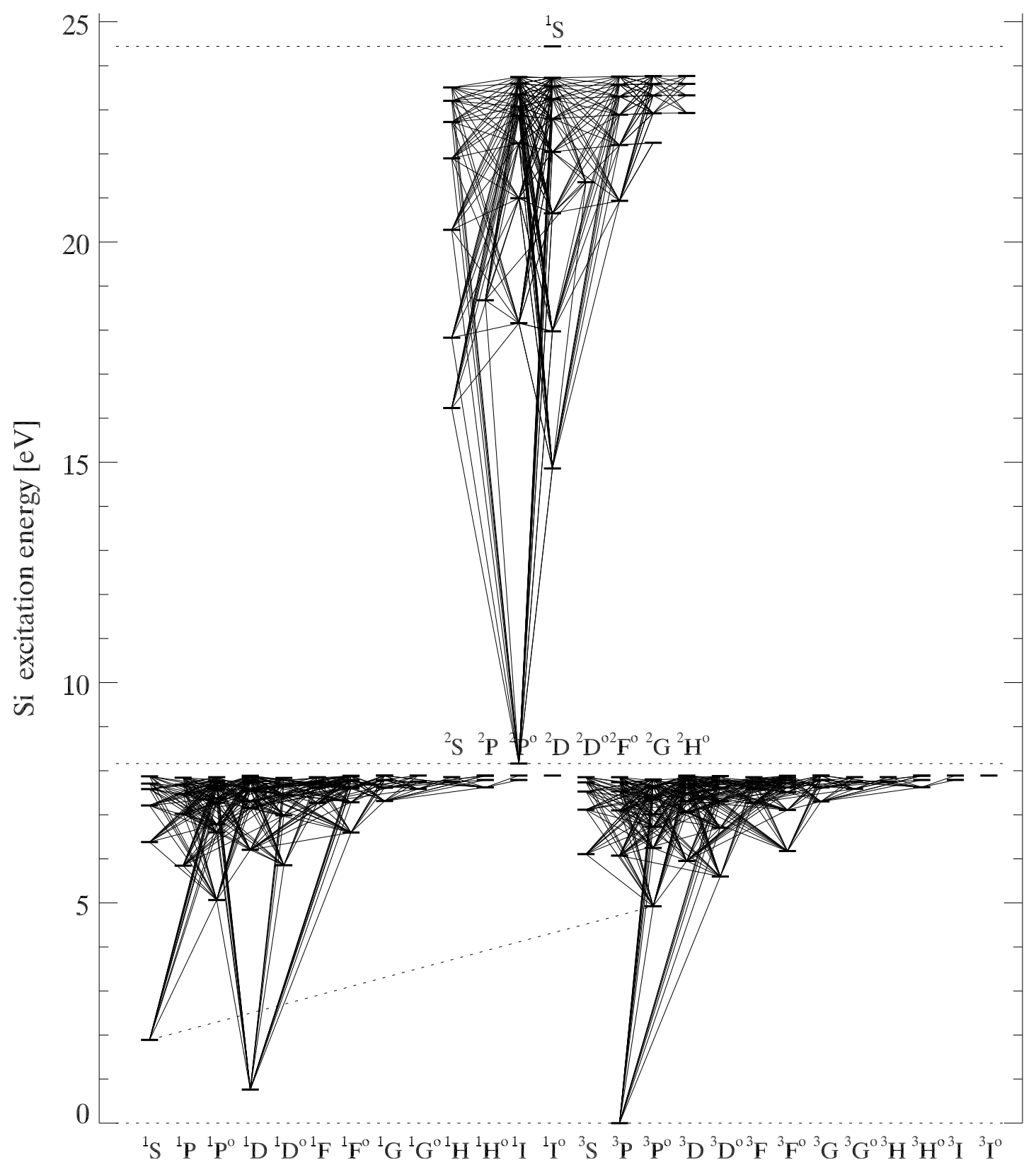

Fig. 1. Term energy diagram of the silicon model atom used in this analysis with Si II (top) and Si I (bottom). Si II quartets are neglected. Allowed transitions are continuous, the forbidden Si I $4103 \AA$ Aline is dotted.

satellite UV, they cannot be observed from the ground. The few near-infrared lines are absorbed from levels above $5 \mathrm{eV}$ and also become invisible in the spectra of metal-poor turnoff stars.

Whereas the solar spectrum still provides enough absorption lines for a thorough abundances analysis, this is not the case for hot metal-poor stars. Even the $4103 \AA$ Antercombination line cannot be used there for an abundance analysis, and silicon abundances of such stars will have to rely on a single line, $\lambda 3905 \AA$. This makes an LTE abundance analysis particularly prone to systematic errors due to atomic interaction processes that tend towards a net over- or underpopulation of the ground state. As we shall see below, the $3 \mathrm{p}^{3} \mathrm{P}_{0,1,2}$ levels and the metastable $3 \mathrm{p}^{1} \mathrm{D}_{2}$ and $3 \mathrm{p}^{1} \mathrm{~S}_{0}$ levels are strongly depopulated by photoionisation, and a NLTE analysis of the line formation problem is therefore unavoidable.
As mentioned above, silicon spectra of cool stars were not investigated often. Only one NLTE analysis has appeared to date. Wedemeyer (2001) reported the influence of deviations from LTE upon the Si abundances in the Sun and in Vega with no reference to line formation in metal-poor stars. A number of LTE analyses, however, cover the whole range of metal abundances, from solar to the most extreme metal-poor halo stars. Among them are the classical analysis of solar silicon lines by Holweger (1973) and the more recent work of Asplund (2000), who introduces 3D line formation based on a solar hydrodynamical model.

The introduction of the atomic model used is the topic of Sect. 2. In Sect. 3 two atmospheric models of the Sun are presented. Section 4 shows the results of the NLTE calculations obtained with different settings of the remaining free parameters. In Sect. 5 spectral line synthesis under both LTE and NLTE assumption is applied to determine the photospheric solar $\mathrm{Si}$ 

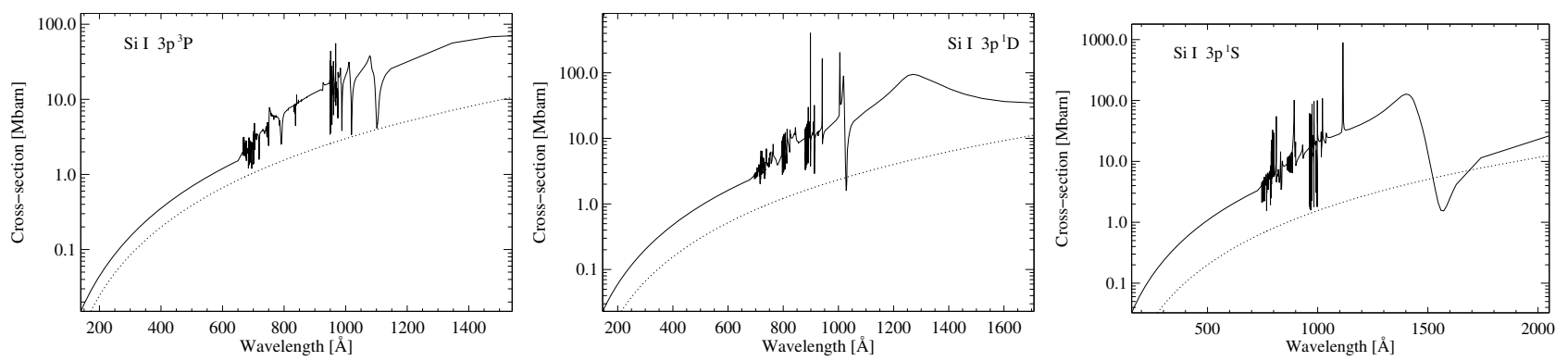

Fig. 2. Photoionisation cross-sections of the metastable terms of the Si I model atom as given in the TOPbase. All three cross-sections are significantly larger than the corresponding hydrogenic approximations (dotted curves). The absorption edge is located at the right boundary of the individual frames.

abundance and the Si I/Si II ionisation equilibrium. The results are discussed in the final section.

\section{Atomic model of silicon}

As usual, the level system of silicon was extracted from the NIST data bank ${ }^{1}$. Silicon in cool stars is fully represented by the first three ionisation stages, where for the purpose of NLTE calculations $\mathrm{Si}$ III reduces to the ground state, $3 \mathrm{~s}^{2}{ }^{1} \mathrm{~S}$. The configuration of the neutral atom includes 4 electrons, with that of the lowest energy being $3 s^{2} 3 p^{2}$. This gives rise to singlets and triplets with a different outer electron replacing $3 \mathrm{p}$. A single quintet term is found near $4 \mathrm{eV}$, which is due to a $3 \mathrm{~s} 3 \mathrm{p}^{3}$ configuration, and which does not couple to any other singlet or triplet term with known line strength, except for the $3 \mathrm{p}^{3} \mathrm{P}-3 \mathrm{p}^{3} \mathrm{~S}^{\mathrm{o}}$. This transition is so weak that the quintet term can safely be neglected. A similar configuration exists in ionised silicon, where the quartet term $3 \mathrm{~s} 3 \mathrm{p}^{2}{ }^{4} \mathrm{P}$ at $5.3 \mathrm{eV}$ is ignored. Our silicon atomic system therefore consists only of the singlet/triplet system of Si I, the doublet system of Si II, and the singlet ground state of Si III. It is shown in Fig. 1 as an energy level diagram with all the terms included here. To keep the number of lines and levels manageable, the level system was reduced to terms implying a thermal differential distribution of the corresponding level populations. Thus, a total of 132 terms of Si I, 41 terms of Si II plus the Si III ground state give our approach a realistic background. As with other elements, completeness is lost at high excitation energies. This holds in particular for the $\mathrm{Si} I \mathrm{II}$ ion, where the last $\mathrm{eV}$ is not represented. However, since these terms are more than $15 \mathrm{eV}$ from the Si II ground state, the population of the levels is extremely low.

Line transitions are based on the work of the Opacity Project (1994), in particular the calculations of Nahar \& Pradhan (1993). The total number of lines included is 786 for Si I and 182 for Si II. For NLTE transfer calculations simple Gauss profiles were used with 9 wavelength points each.

Fortunately, bound-free transition cross-sections with complex structure are available from calculations at the TOPbase ${ }^{2}$ (see also Nahar \& Pradhan 1993). The cross-sections of the lowest three terms, $3 p^{3} \mathrm{P}, 3 \mathrm{p}{ }^{1} \mathrm{D}$ and $3 \mathrm{p}^{1} \mathrm{~S}$ are an order of magnitude greater than those of the next terms, which is very similar to the configuration of $\mathrm{Mg}$ (Zhao et al. 1998). It tends to decouple the metastable terms efficiently from the excited ones.

Background opacities are calculated with an opacity sampling code based on the hydrogen lines, the line lists made available by Kurucz (1992), and on the important bound-free

\footnotetext{
1 http://www.physics.nist.gov/

${ }^{2}$ http://vizier.u-strasbg.fr/topbase/topbase.html
}

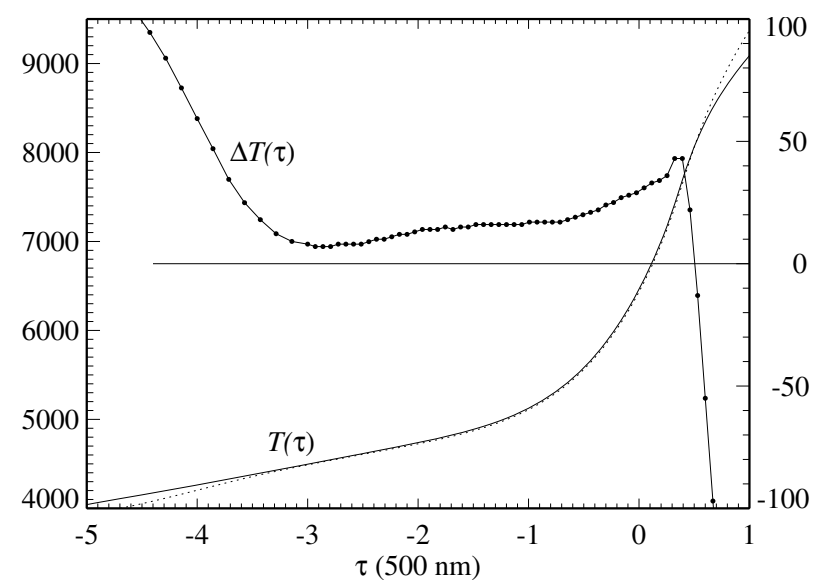

Fig. 3. Temperature stratifications of ODF (dotted) and OS (solid line) solar models (left axis). Difference between the two stratifications, $\Delta T(\tau)=T(\tau) \mathrm{OS}-T(\tau) \mathrm{ODF}$ (right axis).

cross-sections of hydrogen and the most abundant metals. The background opacities are sampled on a random grid of between 5000 and 10000 wavelengths, to which are added the wavelengths of the line profiles. The final NLTE line formation program thus samples roughly 14000 wavelengths. In the UV, where most of the background opacity is expected, the wavelength intervals of the random grid are between 1 and $4 \AA$. We emphasise that the choice of this frequency grid affects only the bound-free radiative interaction rates. Earlier experiments with a finer frequency mesh of up to 40000 randomly sampled frequencies for Fe I (Gehren et al. 2001) showed that the influence of a very dense frequency sampling is less than marginal. This is similar to the sampling situation for OS model atmospheres, where changes in temperature corrections drop below a few $\mathrm{K}$ when the number of frequencies is increased to values beyond 10000 (Grupp 2004, Fig. 8). Background opacities for boundbound transitions are sampled at the corresponding line frequencies.

For our Si NLTE calculations we take into account inelastic collisions with electrons and hydrogen atoms for both excitation and ionisation. The formulae of van Regemorter (1962) and Allen (1973) are used to describe the excitation of allowed and forbidden transitions by electron collisions, respectively. Ionisation cross-sections for electron collisions are calculated applying the formula of Seaton (1962). Drawin's (1968, 1969) formula as described by Steenbock \& Holweger (1984) is used to calculate neutral hydrogen collisions, with a similar formula for bound-free hydrogen collisions. To allow for some empirical correction to the Drawin approximations, a scaling factor $S_{\mathrm{H}}$ is applied to the formula in our calculations. 


\section{Solar model atmospheres}

Solar atmosphere models are supposed to give the basic background for the NLTE line formation program as well as to the spectrum synthesis. This investigation assumes a standard 1D model atmosphere in radiative-convective equilibrium, where convection is treated in a mixing-length approach. Since there exist two different opacity approximations based either on opacity distribution functions (ODF) or on opacity sampling (OS), it seems worthwhile extending our research to the differences of the silicon level populations between the two atmospheric models.

For this purpose both solar models are considered. The ODF model is our old MAFAGS standard atmosphere, nearly identical to that of Kurucz (1992), but downscaled from Kurucz' value of $\log \varepsilon_{\mathrm{Fe}}=7.67$ to a solar Fe abundance of $\log \varepsilon_{\mathrm{Fe}}=7.51$. It was used in all previous NLTE calculations (e.g. Gehren et al. 2001). The OS model is based on a similar model atmosphere code of Grupp (2004), but with convection treated according to Canuto \& Mazzitelli (1992), and with opacities sampled from continuous opacities and from an extended line list of Kurucz $\&$ Bell (1995) at 86000 randomly chosen frequency points. The two types of atmospheres lead to slightly different temperature stratifications, as can be seen in Fig. 3. The two models differ in temperature outside $\log \tau \simeq 0.3$ with a moderately increasing amplitude. Note that the differences outside $\log \tau=-3$ do not affect the observed Si spectrum, whereas inside $\log \tau=0.3$ the gap between the temperature stratifications is the result of different versions of the mixing-length theory (see Grupp 2004, for a more detailed comparison). The latter marginally affects the formation of the Balmer lines, but does not influence Si line formation very much.

\section{NLTE line formation results}

The Si departure coefficients for the more important terms of Si I and Si II shown for our final selection of the hydrogen collision parameter $\left(S_{\mathrm{H}}=0.1\right)$ in Fig. 4 are surprising in that they follow LTE out into the middle photosphere. This has not been encountered in any of the other neutral ions analysed so far. It is the result of an unusually large energy gap between the ground state, $3 p^{3} \mathrm{P}$, and the two metastable terms $3 p^{1} \mathrm{D}$, and $3 \mathrm{p}^{1} \mathrm{~S}$, on one side, and the first excited levels of neutral silicon on the other. These gaps are $\sim 5,4$, and $3 \mathrm{eV}$, respectively, and they shift all lines emerging from those levels into the UV, most of them below $2000 \AA$, where the Al I absorption edge is suppressed the radiation field by an order of magnitude. As a result, all these strong ${ }^{3}$ lines have only weak radiative rates due to a very low integrated mean radiation field. There is a marginal similarity with the Mg I ion (Zhao et al. 1998), however, with the difference that the more important low-excitation transitions in neutral magnesium are well above $2000 \AA$ and thus considerably stronger. This is also the case for Fe I which shares ionisation energy and solar abundance with both $\mathrm{Mg}$ and $\mathrm{Si}$ but has a much more complex line spectrum with strong lines in the visible.

Therefore, the interaction of the three most populated Si I levels with the Si II ion is completely based on bound-free processes, where photoionisation and ionisation by electron collisions compete in strength, with a small net pumping to the Si II ground state. As would be expected, the $3 p^{1} \mathrm{D}$ and $3 \mathrm{p}^{1} \mathrm{~S}$ terms follow $3 \mathrm{p}^{3} \mathrm{P}$ tightly. In the absence of stronger radiative rates

\footnotetext{
3 According to their population and $f$-value.
}
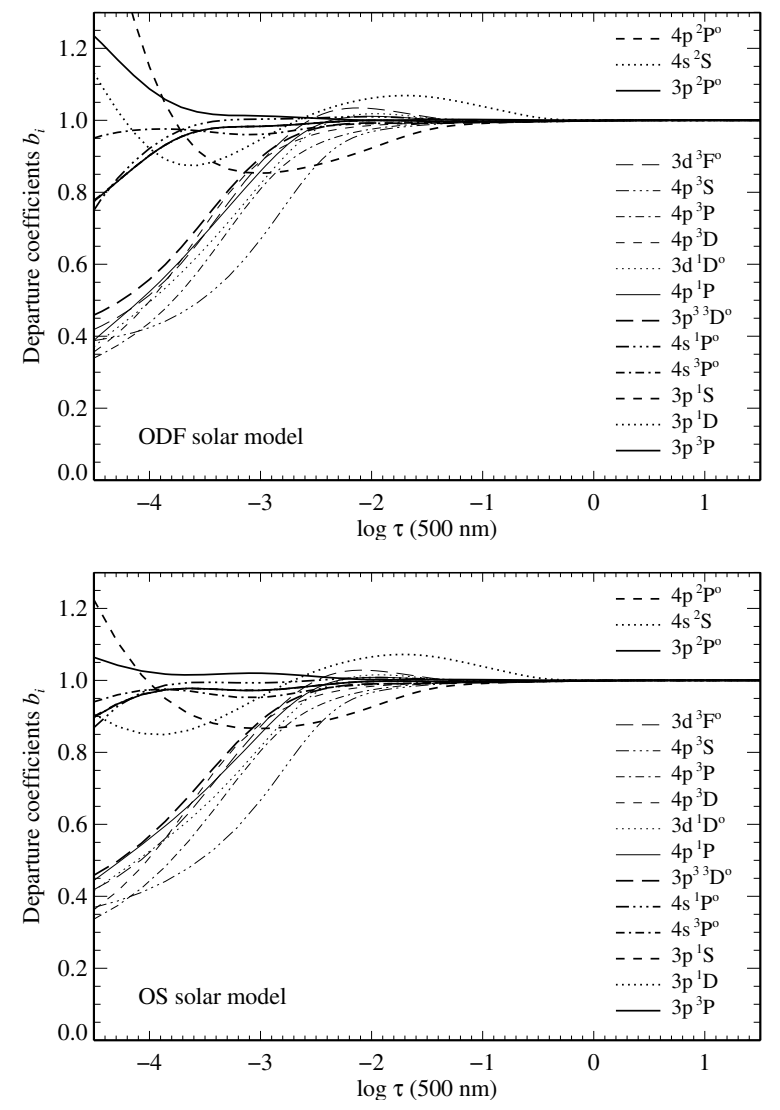

Fig. 4. Departure coefficients $b i=\mathrm{N} i / \mathrm{N} i^{\mathrm{LTE}}$ for the final parametrization of the Si atomic model $\left(S_{\mathrm{H}}=0.1\right)$ calculated for an ODF (top) and for an OS (bottom) solar model atmosphere as function of the standard optical depth. Note the similarity of the results for both atmospheric models. The doublet terms $4 \mathrm{~s}^{2} \mathrm{~S}, 4 \mathrm{p}^{2} \mathrm{P}^{\mathrm{o}}$ belong to Si II.

the excited terms are determined by collisions in their respective regions of line formation.

Test calculations applying increased photoionisation or decreased electronic collisions reveal that $\mathrm{Si}$ I is relatively stable against a variation of atomic data. Although a strongly increased photoionisation (see Fig. 5, top) starts to depopulate the levels already in deeper layers, this does not affect neutral Si line formation very much, because most of the lines observed in the visible and near infrared solar spectrum are weak and thus formed in deeper layers. The only strong line at $3905 \AA$ retains a thermalised source function out to the upper photosphere. The ionisation itself changes only by a very small fraction. The bottom of Fig. 5 demonstrates a variation of the electron collision rates by a factor of 0.1. Similar to the increased photoionisation, though less pronounced in its influence on the departure coefficients, the reduction of the electronic collision efficiency also leads only to a marginal de-thermalisation, that does not affect neutral silicon line formation very much. Only the near-infrared lines respond to collisions (see below).

While the Si II $3 \mathrm{p}^{2} \mathrm{P}^{\mathrm{o}}$ ground state is a true mirror of the $\mathrm{Si}$ I $3 \mathrm{p}^{3} \mathrm{P}$ ground state, the excited levels of Si II lead to absorption lines coming from much deeper layers in the atmosphere. This holds in particular for the highly excited $4 \mathrm{~s}^{2} \mathrm{~S}-4 \mathrm{p}^{2} \mathrm{P}^{\mathrm{o}}$ transition with lines at 6347.095 and $6371.360 \AA$, respectively. Although absorbed at an excitation energy of more than $8 \mathrm{eV}$, the lines are clearly visible in the spectra of solar-type stars and may therefore serve as a test for the ionisation and $\mathrm{Si}$ II excitation equilibrium in the Sun. 

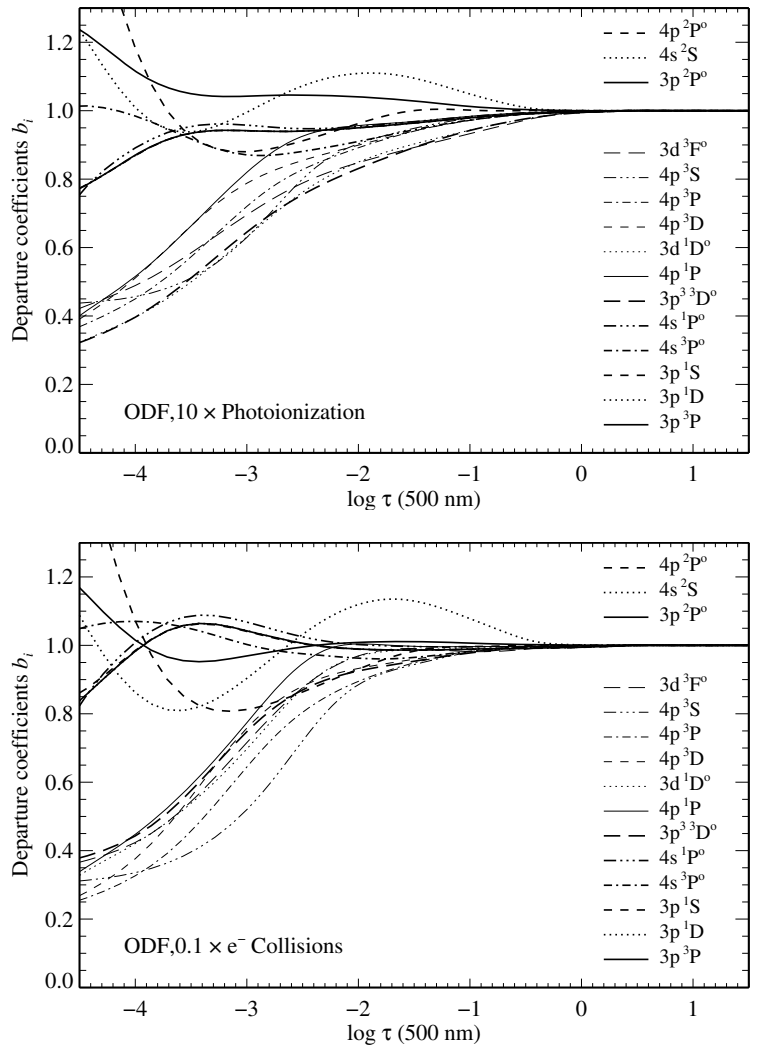

Fig. 5. Departure coefficients for the final atomic model in the ODF solar atmosphere. All parameters are the same as in Fig. 4, except that the photoionisation rates were scaled by a factor of 10 (top), and the electronic collision rates divided by a factor of 10 (bottom).

A variation of the hydrogen collision scaling factor $S_{\mathrm{H}}$ between 0 and 1 allows us to introduce similar changes as those resulting from electron collisions. Due to the large number of hydrogen atoms, the neutral hydrogen collision rates can be significantly stronger than the electron collision rates. Yet, in the range of $S_{\mathrm{H}}$ parameters considered here, they do not lead to significant changes in the line profiles in the visible where, generally, abundance differences for the two extremes $\left(S_{\mathrm{H}}=0\right.$ and 1$)$ stay within $0.03 \ldots 0.04$ dex. This situation changes significantly, whenever the Si I near-infrared lines are analysed. As is evident from Table 1 most of these are absorbed from either $4 s^{3} \mathrm{P}^{\mathrm{o}}, 4 \mathrm{p}{ }^{1} \mathrm{P}$, or $4 \mathrm{p}^{3} \mathrm{D}$. Since all the excited terms are only loosely coupled to each other and to other high-excitation terms, their departure coefficients tend to diverge from each other at $\log \tau \simeq-2$, i.e. where the cores of the relatively strong near-infrared lines form. This is the reason for a slightly increased abundance trend going with the hydrogen collision scaling factor. For lines beyond $1 \mu \mathrm{m}$ the corresponding abundance variations can reach more than 0.1 dex, and their cores suggest the choice of $S_{\mathrm{H}}=0.1$.

All calculations were carried out with a revised version of the DETAIL program (Butler \& Giddings 1985) using accelerated lambda iteration following the extremely efficient method described by Rybicki \& Hummer (1991, 1992).

\section{Line synthesis and the solar Si abundance}

The observed solar flux spectrum was taken from the Kitt Peak Atlas (Kurucz et al. 1984). Spectral line synthesis is performed interactively using the SIU program package of Reetz (1991). The profiles of all the lines given in Table 1 were calculated for various assumptions, with atomic parameters varied as described above. Van der Waals damping constants $\log C_{6}$ for Si I lines were computed according to the Anstee \& O'Mara (1991, 1995) interpolation tables. For use in other stars the $\log g f \varepsilon_{\odot}$ values in the last column are most important. They are given in Table 1 for our final atomic model statistical equilibrium calculations with $S_{\mathrm{H}}=0.1$, and they are used to determine solar Si abundances below. Van der Waals broadening constants for the Si II lines are purely empirical allowing an optimal fit of the line profiles. Since the profiles are simultaneously affected by macroturbulence broadening, similar fits could be obtained also with $\sim 5$ times smaller damping constants. Due to this bias the resulting abundances are uncertain by 0.05 dex.

Figure 6 shows some fits of the near-infrared lines between 1.0 and $1.3 \mu \mathrm{m}$. In contrast to the $\mathrm{Si}$ lines in the visible, the cores of the near-infrared lines deviate from the LTE profiles. It is only this variation with the degree of thermalisation that allows us to determine a best fit value of $S_{\mathrm{H}}=0.1$. As mentioned above, this selection does not affect the visible line profiles.

Nearly all of the Si line profiles observed in the solar spectrum display some kinematic asymmetry with a red-shifted line core compared to the fits shown in Fig. 6 . This difference does not depend on line blends, for some of which information is evidently missing. The effect is accompanied in most lines by a corresponding blue shift of the blue line wings, and sometimes by observed line wings that are both deeper than any profile fit possible under line synthesis with the currently adopted solar parameters. We note that a large part of such defects are the result of our approximate handling of photospheric velocity fields. In a 1D analysis of spectral line formation, all known atmospheric motions are replaced by a micro-/macroturbulence approach that can at best account for a mean velocity field. A $3 \mathrm{D}$ analysis would probably remove most of these shortcomings (see Asplund 2005, for a review). Modelling the complex granular velocities with a $1 \mathrm{D}$ model, however, would at least require the macroturbulence velocities $\Xi$ to be adjusted to the regions of line formation, which are different for weak and strong lines. This is impossible with a single macroturbulence convolution, because higher $\Xi$ values would be needed for the weak lines than for the strong line wings.

When it comes to spectral line synthesis, every effort to determine the absolute solar photospheric silicon abundance is hampered by uncertain or inconsistent $f$-values. Since the lifetime experiments of Savage \& Lawrence (1966) and Marek \& Richter (1973) there has been an ongoing discussion of the measurements regarding the lifetime of the Si I $4 s^{3} \mathrm{P}_{2}^{\mathrm{o}}$ level. While all other level lifetimes led to consistent results, this showed controversial values depending on the experimental method used (Bashkin et al. 1980; Becker et al. 1980a,b; Smith et al. 1987). The lifetime measurements of the $4 \mathrm{~s}^{3} \mathrm{P}_{2}^{\mathrm{o}}$ level were carried out with three methods: phase shift, beam foil, and laser fluorescence, of which the last, used by Becker et al. (1980a), should be the most reliable. However, this value, $\tau=4.4 \pm 0.4 \mathrm{~ns}$ is at variance with the four independent lifetime results obtained by the other authors, $6.8 \pm 0.8,5.95 \pm 0.48,5.9 \pm 0.7$ and $6.3 \pm 0.6 \mathrm{~ns}$, respectively. Thus the correspondingly calibrated absolute $f$-values differ by roughly $25 \%$, which translates into a systematic abundance difference of 0.10 dex. This is still the current systematic uncertainty of the photospheric solar silicon abundance.

To determine the solar $\mathrm{Si}$ abundance, it is also necessary to judge the available $f$-values according to their consistency. In Fig. 7 both the Kurucz and the NIST $f$-values produce an enormous abundance scatter with standard deviations of $\sigma\left(\log \varepsilon_{\odot}\right)=$ 0.40 and 0.38 , respectively. On the other hand, the relative 

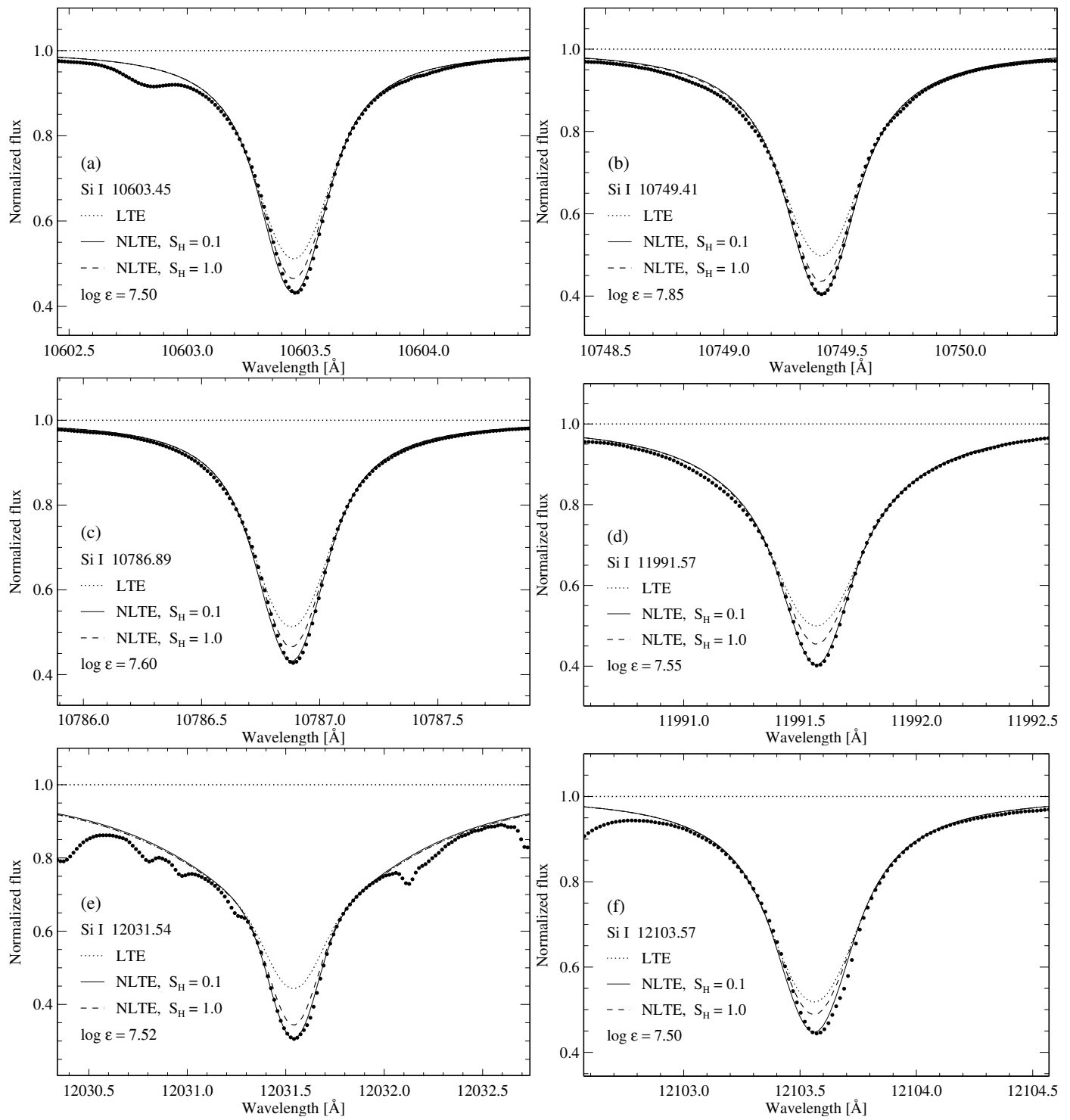

Fig. 6. Near-infrared Si I solar line profiles. NLTE and LTE profiles are calculated for the same abundance, where the NLTE profile with $S_{\mathrm{H}}=0.1$ refers to the best fit.

$f$-values of Garz (1973) are still the only source for high-quality data of lines in the visible, with $\log \varepsilon_{\odot}(\mathrm{Garz})=7.62 \pm 0.05$. None of the sets of oscillator strengths displays a clear trend in either of the different parameter ranges, although it may be significant that the three most deviating abundances, based on Kurucz \& Peytremann's oscillator strengths, emerge from the $4 \mathrm{~s}^{3} \mathrm{P}^{\mathrm{o}}$ term, i.e. the term for which the lifetime measurements were most contradictory. Removing five outlyers of the NIST abundances from the sample, the resulting scatter about the mean abundance is significantly reduced, now with $\log \varepsilon_{\odot}(\mathrm{NIST})=$ $7.52 \pm 0.13$.

As seen in Table 1, it is possible in the solar spectrum to measure the line profiles absorbed from the Si II $4 s^{2} \mathrm{~S}_{1 / 2}$ level, which is excited to more than $8 \mathrm{eV}$. The results are shown in Fig. 8; the line data and $\mathrm{Si}$ abundances are found in Table 1 and Fig. 7, respectively. Both LTE and NLTE give the same abundances within 0.01 dex. Although not perfect, the ionisation equilibrium, i.e. the abundance difference between Si II and Si I (using Garz' $f$-values) is $\simeq-0.08$ dex, just at the limit of a $2 \sigma$ interval. It is important to note that the two lines depend sensitively on the choice of the damping constant, represented here by $C_{6}$. Using Kurucz' van der Waals broadening constant which is more than a factor 10 smaller, and replacing the opacity difference by slightly stronger blends, implies a change in the abundances by $\simeq 0.05 \ldots 0.10 \mathrm{dex}$. Thus the Si II abundances add to the results of the neutral data, but they are by no means decisive.

Altogether, applying the laser fluorescence lifetime measurements to the Garz $f$-values would increase the latter by 0.1 dex and thus reduce the abundance by the same amount. The resulting common mean abundance together with the NIST values would be $\log \varepsilon_{\odot}=7.52 \pm 0.09$. This would include the Si II lines and lead to a perfect ionisation equilibrium.

The influence of NLTE and of the choice of the model atmosphere on the solar abundances is best viewed using the line wavelength as an $x$-axis. Here we present in Fig. 9a the logarithmic abundance differences between NLTE (final atomic model, $\left.S_{\mathrm{H}}=0.1\right)$ and LTE, both from profile fits using the ODF solar model. Although the mean abundance difference is small, the systematic increase towards near-infrared wavelengths is remarkable. The lines with low NLTE abundances all have relatively strong equivalent widths between 100 and $350 \mathrm{m \AA}$. Their 

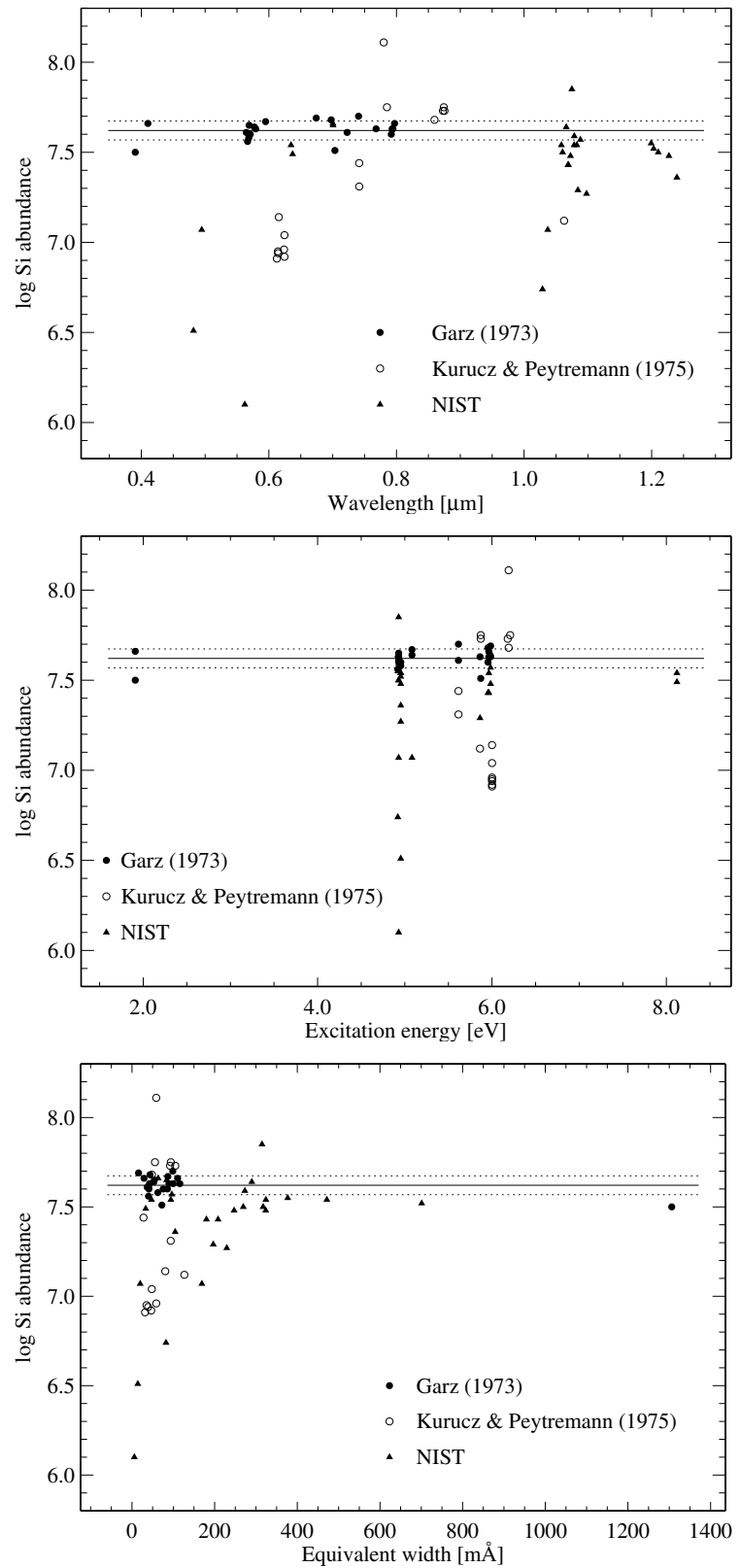

Fig. 7. Solar abundances calculated with three different sets of $f$-values. The mean value is shown as a straight line only for the Garz (1973) data, together with the dotted $\pm 1 \sigma$ error lines.

$f$-values are much stronger than those of the visual lines that arise from the same lower levels, mostly $4 \mathrm{~s}^{3} \mathrm{P}^{\mathrm{o}}, 4 \mathrm{~s}^{1} \mathrm{P}^{\mathrm{o}}$, and $4 p^{3} \mathrm{D}$, because the visual lines lead to even higher excited levels (see Fig. 1). While most of the visible lines are formed well inside $\log \tau_{5000}=-2$, the region of line formation for the near-infrared lines is shifted outward to $\log \tau_{5000}=-3 \ldots-2$, where the line source function of those lines differs significantly from the local Planck function. For the strongest nearinfrared lines, however, the core contributes only marginally to the abundance determination, which is dominated by the strong line profile wings coming from a line formation region inside $\log \tau_{5000}=-2$. Therefore, the trend in Fig. 9a documents the different line saturation (see also Fig. 6).

Figure $9 \mathrm{~b}$ displays the abundance differences calculated for the final NLTE models between the ODF and the OS solar atmosphere. Here, we note a similar trend with wavelength, but the corresponding correlation with the strong lines is much less
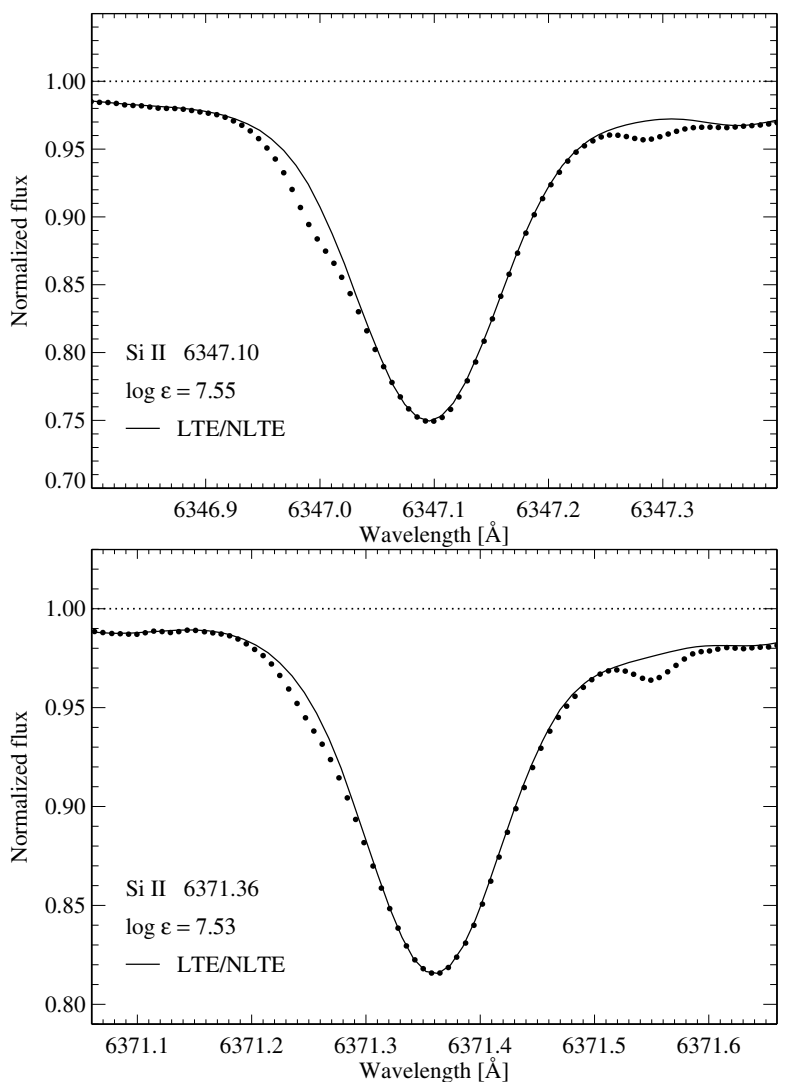

Fig. 8. Solar profile fits for highly excited Si II lines at 6347 and $6371 \AA$ A. Blend calculations do not include terrestrial atmospheric lines.

pronounced. It is more likely that the different photospheric temperature gradients (see Fig. 3) contribute to the change in the abundance differences, because the weaker $\mathrm{Si}$ I lines in the visible are formed at $50 \mathrm{~K}$ higher temperatures and thus require a slightly higher abundance. The mean $\mathrm{Si}$ abundance does not depend on the choice of the model atmosphere.

\section{Discussion}

Both the atomic model and the line formation results do not differ substantially from the previous analysis of Wedemeyer (2001). Our aims of atomic model completeness were more directed towards inclusion of high energy levels, whereas Wedemeyer apparently favoured level completeness up to a limiting energy. Therefore, he included a Si I quintet and some Si II quartet terms, all of which are coupled only by weak forbidden transitions to the leading multiplets. In fact, our test calculations show that these terms are insignificant for the departure coefficients.

Therefore it is not too surprising that our results agree in many details. Most important is our agreement with respect to the nearly thermal population of all Si I levels inside optical depth $\log \tau_{5000}=-2$. In fact, Wedemeyer's departure coefficients (see his Fig. 2) are even more thermalised than ours. The remaining differences probably come from slightly different interaction cross-sections. Our solar silicon abundances are also very similar, except for an offset that is mostly due to his adjustment of the Garz (1973) oscillator strengths to the Becker et al. (1980a,b) lifetime measurement. We also agree with his result that in the solar photosphere $\mathrm{Si}$ abundance corrections due to NLTE departures are small. However, it must be noted that such 

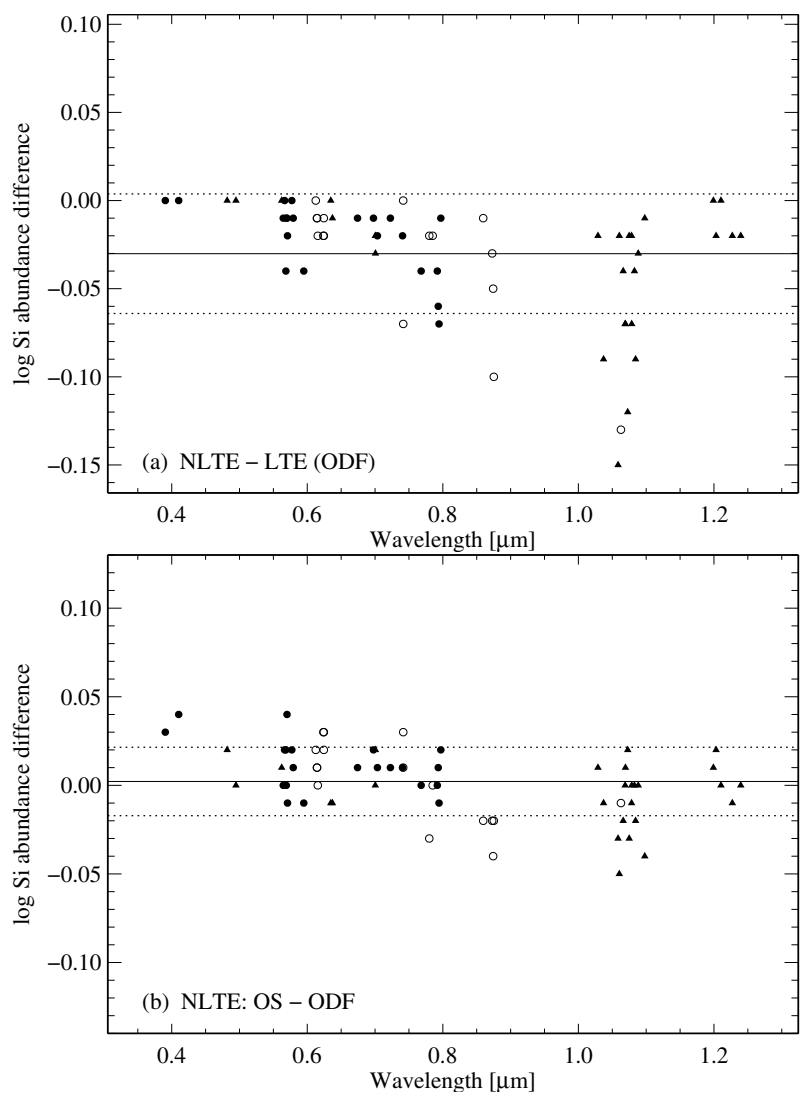

Fig. 9. Logarithmic solar abundance differences for silicon lines.

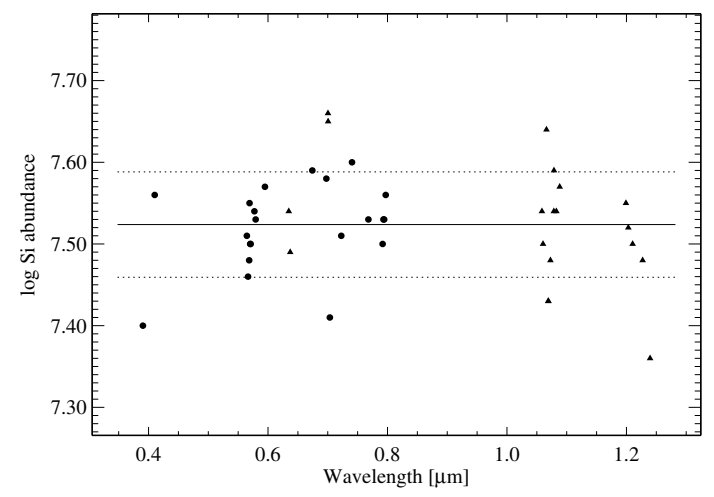

Fig. 10. Corrected logarithmic solar Si abundances with $f$-values from Garz (1973, filled circles, corrected by +0.1 ) or from NIST (filled triangles, omitting eight outlyers). See text for discussion.

abundance corrections become significant in the near infrared, where the cores of strong lines are important.

As is the case for all elements, the agreement between meteoritic abundances and those obtained from photospheric analysis is a major topic of research. Because the solar photospheric silicon abundance is used as a reference for $\alpha$-elements in the Galaxy, and as the normalising element for meteoritic abundances in the solar system, it is even more important for the early chemical evolution of the Milky Way. In particular the question arises as to whether the meteoritic and photospheric $\mathrm{Si} / \mathrm{Fe}$ ratios are the same. Figure 10 combines the selected removal of eight lines with deviating NIST $f$-values with the correction of the Garz $f$-values for a $25 \%$ smaller lifetime of the Si I $4 \mathrm{~s}^{3} \mathrm{P}_{2}^{\mathrm{o}}$ level as measured by Becker et al. (1980a). This brings together the blue, visual and near-infrared wavelength region on a common abundance scale and avoids excessive scatter. The resulting photospheric abundance, $\log \varepsilon_{\odot}$ (cor) $=7.52 \pm 0.06$ is in good agreement with the results of Asplund (2000) and Wedemeyer (2001).

During the last years it has become evident that 1D abundance analyses carry systematic errors as compared with 3D results. While the 3D results are only a few, and their reliability is not yet beyond doubt, it seems that some of the metals analysed in 3D or 2D lead to slightly different photospheric abundances. One of the main obstacles against a full hydrodynamic investigation is still the limits of computing speed and memory, respectively. Yet preliminary calculations cited by Wedemeyer and the 2D simulations of Steffen \& Holweger (2002) indicate solar abundance corrections for weak Si I lines of $\simeq+0.02$, whereas Asplund (2000) favors a 3D hydrodynamical correction of $\simeq-0.02$ for his set of Si lines, both with reference to the solar model of Holweger \& Müller (1974). The final abundance thus may be between 7.50 and 7.54. With a meteoritic ratio of $\log \varepsilon_{\mathrm{Si}}-\log \varepsilon_{\mathrm{Fe}}=0.06$ (Lodders 2003; Asplund et al. 2005), the meteoritic Fe abundance would be as low as 7.44 .. 7.48.

Acknowledgements. This research was supported by the National Natural Science Foundation of China under grant Nos. 10433010, 10521001, 10778626 and 1071113000052, the National Basic Research Program of China (973 Program) under grant No. 2007CB815103, and by the Deutsche Forschungsgemeinschaft with grant 446 CHV 112/1,2.

\section{References}

Allen, C. W. 1973, Astrophys. Quantities, 3rd edn. (London: Athlone Press) Anstee, S. D., \& O‘Mara, B. J. 1991, MNRAS, 253, 549

Anstee, S. D., \& O'Mara, B. J. 1995, MNRAS, 276, 859

Asplund, M. 2000, A\&A, 359, 755

Asplund, M. 2005, ARA\&A, 43, 481

Asplund, M., Grevesse, N., \& Sauval, A. J. 2005, ASP Conf. Ser., 336, 25

Bashkin, S., Astner, G., Mannervik, S., et al. 1980, Phys. Scr., 21, 820

Becker, U., Zimmermann, P., \& Holweger, H. 1980a, Geochim. Cosmochim. Acta, 44, 2145

Becker, U., Kerkhoff, H., Kwiatkowski, M., et al. 1980b, Phys. Lett. 76A, 125 Butler, K., \& Giddings, J. 1985, Newsletter on the analysis of astronomical spectra No. 9, University of London

Canuto, V. M., \& Mazzitelli, I. 1992, ApJ, 389, 724

Drawin, H. W. 1968, Z., Phys., 211, 404

Drawin, H. W. 1969, Z., Phys., 225, 483

Garz, T. 1973, A\&A, 26, 471

Gehren, T., Butler, K., Mashonkina, L., Reetz, J., \& Shi, J. R. 2001, A\&A, 366, 981

Grupp, F. 2004, A\&A, 420, 289

Holweger H. 1973, A\&A, 26, 275

Holweger H., \& Müller, E. A. 1974, Sol. Phys. 39, 19

Kurucz, R. L. 1992, Rev. Mex. Astron. Astrofis. 23, 45

Kurucz, R. L., \& Bell, B. 1995, Atomic Line Data, Kurucz CD-ROM No. 23, Cambridge, Mass.: Smithsonian Astrophysical Observatory

Kurucz, R. L., \& Peytremann, E. 1975, A table of semiempirical gf-values, SAO Spec.Rep. No. 362, Cambridge, Mass

Kurucz, R. L., Furenlid, I., Brault, J., et al. 1984, Solar Flux Atlas from 296 to 1300 nm, Kitt Peak National Solar Observatory

Lodders, K. 2003, ApJ, 591, 1220

Marek, J., \& Richter, J. 1973, A\&A, 26, 155

Nahar, S. N., \& Pradhan, A. K. 1993, J. Phys. B, 26, 1109

Reetz, J. K. 1991, Diploma Thesis, Universität München

Rybicki, G. B., \& Hummer, D. G. 1991, A\&A, 245, 171

Rybicki, G. B., \& Hummer, D. G. 1992, A\&A, 262, 209

Savage, B. D., \& Lawrence, G. M. 1966, ApJ, 146, 940

Seaton, M. J. 1962, in Atomic and Molecular Processes (New York: Acad. Press)

Seaton, M. J., Mihalas, D., \& Pradhan, A. K. 1994, MNRAS, 266, 805

Smith, P. L., Huber, M. C.E., Tozzi, G. P., et al. 1987, ApJ 322, 573

Steenbock, W., \& Holweger, H. 1984, A\&A, 130, 319

Steffen, M., \& Holweger, H. 2002, A\&A, 387, 258

van Regemorter, H. 1962, ApJ, 136, 906

Wedemeyer, S. 2001, A\&A, 373, 998

Zhao, G., Butler, K., \& Gehren, T. 1998, A\&A, 333, 219 
J. R. Shi et al.: Statistical equilibrium of silicon in the solar atmosphere, Online Material $p 1$

Table 1. Atomic data for spectral lines of Si I and Si II (last two entries). References to the $g f$-values are G: Garz (1973, experimental), K: Kurucz \& Peytremann (1975), and N: NIST (http://wWW . physics.nist.gov/). Values for $\log g f \varepsilon_{\odot}$ refer to our final atomic model, with $S_{\mathrm{H}}=0.1$.

\begin{tabular}{|c|c|c|c|c|c|c|}
\hline Mult & $\begin{array}{c}\lambda \\
{[\AA]}\end{array}$ & Line & $\begin{array}{c}E_{\text {low }} \\
{[\mathrm{eV}]}\end{array}$ & $\log C_{6}$ & $\log g f$ & $\begin{array}{c}\log \\
g f \varepsilon_{\odot}\end{array}$ \\
\hline 2 & 4102.93 & $3 p^{1} S_{0}-4 s^{3} P_{1}^{o}$ & 1.909 & -30.972 & $-3.14 \mathrm{G}$ & 4.52 \\
\hline 3 & 3905.53 & $3 \mathrm{p}^{1} \mathrm{~S}_{0}-4 \mathrm{~s}^{1} \mathrm{P}_{1}^{\mathrm{o}}$ & 1.909 & -30.917 & $-1.09 \mathrm{G}$ & 6.41 \\
\hline \multirow[t]{5}{*}{4} & 12103.56 & $4 s^{3} P_{1}^{o}-4 p^{3} D_{1}$ & 4.707 & -30.759 & $-0.35 \mathrm{~N}$ & 7.15 \\
\hline & 11991.57 & $4 s^{3} P_{0}^{0}-4 p^{3} D_{1}^{1}$ & 4.920 & -30.760 & $-0.29 \mathrm{~N}$ & 7.26 \\
\hline & 12031.54 & $4 s^{3} P_{2}^{0}-4 p^{3} D_{3}$ & 4.954 & -30.743 & $0.41 \mathrm{~N}$ & 7.93 \\
\hline & 12270.72 & $4 s^{3} P_{2}^{2}-4 p^{3} D_{2}^{3}$ & 4.954 & -30.752 & $-0.35 \mathrm{~N}$ & 7.13 \\
\hline & 12395.85 & $4 s^{3} P_{2}^{o}-4 p^{3} D_{1}^{2}$ & 4.954 & -30.757 & $-1.53 \mathrm{~N}$ & 5.83 \\
\hline \multirow[t]{6}{*}{5} & 10603.45 & $4 s^{3} P_{1}^{2}-4 p^{3} P_{2}$ & 4.707 & -30.677 & $-0.32 \mathrm{~N}$ & 7.16 \\
\hline & 10749.40 & $4 s^{3} P_{1}^{o}-4 p^{3} P_{1}^{2}$ & 4.707 & -30.689 & $-0.54 \mathrm{~N}$ & 7.30 \\
\hline & 10786.88 & $4 s^{3} P_{1}^{0}-4 p^{3} P_{0}^{1}$ & 4.707 & -30.691 & $-0.42 \mathrm{~N}$ & 7.16 \\
\hline & 10661.00 & $4 s^{3} P_{0}^{0}-4 p^{3} P_{1}^{0}$ & 4.920 & -30.687 & $-0.41 \mathrm{~N}$ & 7.22 \\
\hline & 10827.10 & $4 s^{3} P_{2}^{0}-4 p^{3} P_{2}^{1}$ & 4.954 & -30.677 & $0.17 \mathrm{~N}$ & 7.71 \\
\hline & 10979.34 & $4 s^{3} P_{2}^{2}-4 p^{3} P_{1}^{2}$ & 4.954 & -30.688 & $-0.32 \mathrm{~N}$ & 6.95 \\
\hline \multirow[t]{3}{*}{6} & 10371.30 & $4 s^{3} P_{1}^{2}-4 p^{3} S_{1}^{1}$ & 4.707 & -30.659 & $-0.41 \mathrm{~N}$ & 6.65 \\
\hline & 10288.90 & $4 s^{3} P_{0}^{0}-4 p^{3} S_{1}^{1}$ & 4.920 & -30.661 & $-0.89 \mathrm{~N}$ & 5.85 \\
\hline & 10585.17 & $4 s^{3} P_{2}^{o}-4 p^{3} S_{1}$ & 4.954 & -30.659 & $-0.18 \mathrm{~N}$ & 7.36 \\
\hline 9 & 5793.07 & $4 s^{3} P_{1}^{o}-5 p^{3} D_{2}$ & 4.707 & -30.294 & $-2.06 \mathrm{G}$ & 5.57 \\
\hline \multirow[t]{5}{*}{10} & 5645.61 & $4 s^{3} P_{1}^{o}-5 p^{3} P_{2}^{2}$ & 4.707 & -30.294 & $-2.14 \mathrm{G}$ & 5.47 \\
\hline & 5690.43 & $4 s^{3} \mathrm{P}_{1}^{\mathrm{o}}-5 \mathrm{p}^{3} \mathrm{P}_{1}^{2}$ & 4.707 & -30.294 & $-1.87 \mathrm{G}$ & 5.77 \\
\hline & 5701.11 & $4 s^{3} P_{1}^{o}-5 p^{3} P_{0}$ & 4.707 & -30.294 & $-2.05 \mathrm{G}$ & 5.55 \\
\hline & 5665.55 & $4 s^{3} \mathrm{P}_{0}^{\mathrm{o}}-5 \mathrm{p}^{3} \mathrm{P}_{1}$ & 4.920 & -30.294 & $-2.04 \mathrm{G}$ & 5.51 \\
\hline & 5708.40 & $4 s^{3} \mathrm{P}_{2}^{\mathrm{o}}-5 \mathrm{p}^{3} \mathrm{P}_{2}$ & 4.954 & -30.294 & $-1.47 \mathrm{G}$ & 6.12 \\
\hline \multirow[t]{2}{*}{11} & 5622.23 & $4 s^{3} P_{1}^{2}-5 p^{3} S_{1}^{2}$ & 4.707 & -30.294 & $-1.64 \mathrm{~N}$ & 4.46 \\
\hline & 5684.48 & $4 s^{3} P_{2}^{o}-5 p^{3} S_{1}$ & 4.954 & -30.294 & $-1.65 \mathrm{G}$ & 5.93 \\
\hline 11.04 & 4818.03 & $4 s^{3} P_{2}^{0}-6 p^{3} D_{3}$ & 4.954 & -30.294 & $-1.57 \mathrm{~N}$ & 4.93 \\
\hline 16 & 5948.54 & $4 s^{1} P_{1}^{2}-5 p^{1} D_{2}^{3}$ & 5.082 & -30.629 & $-1.23 \mathrm{G}$ & 6.43 \\
\hline 17 & 5772.15 & $4 s^{1} P_{1}^{o}-5 p^{1} S_{0}^{2}$ & 5.082 & -30.287 & $-1.75 \mathrm{G}$ & 5.89 \\
\hline 17.09 & 4947.61 & $4 s^{1} P_{1}^{o}-6 p^{1} S_{0}$ & 5.082 & -30.287 & $-1.81 \mathrm{~N}$ & 5.26 \\
\hline 22 & 7415.96 & $3 p^{3}{ }^{3} \mathrm{D}_{2}^{\mathrm{o}}-4 \mathrm{f}^{1} \mathrm{~F}_{3}$ & 5.616 & -29.969 & $-0.50 \mathrm{~K}$ & 6.80 \\
\hline 23 & 7405.79 & $3 p^{3}{ }^{3} \mathrm{D}_{1}^{\mathrm{o}}-4 \mathrm{f}^{3} \mathrm{~F}_{2}^{3}$ & 5.614 & -29.869 & $-0.82 \mathrm{G}$ & 6.88 \\
\hline 23 & 7415.36 & $3 p^{3}{ }^{3} \mathrm{D}_{2}^{\mathrm{o}}-4 \mathrm{f}^{3} \mathrm{~F}_{2}$ & 5.616 & -29.869 & $-1.60 \mathrm{~K}$ & 5.84 \\
\hline 26 & 7226.21 & $3 p^{3}{ }^{3} \mathrm{D}_{1}^{\mathrm{o}}-4 \mathrm{f}^{1} \mathrm{D}_{2}^{2}$ & 5.614 & -29.800 & $-1.51 \mathrm{G}$ & 6.09 \\
\hline 27 & 6244.47 & $3 p^{3}{ }^{3} \mathrm{D}_{2}^{\mathrm{o}}-5 \mathrm{f}^{1} \mathrm{D}_{2}$ & 5.616 & -29.868 & $-0.69 \mathrm{~K}$ & 6.22 \\
\hline 28 & 6237.32 & $3 p^{3}{ }^{3} \mathrm{D}_{1}^{\mathrm{o}}-5 \mathrm{f}^{3} \mathrm{~F}_{2}$ & 5.614 & -29.869 & $-0.53 \mathrm{~K}$ & 6.43 \\
\hline 28 & 6243.82 & $3 p^{3}{ }^{3} \mathrm{D}_{2}^{\mathrm{o}}-5 \mathrm{f}^{3} \mathrm{~F}_{3}$ & 5.616 & -29.868 & $-0.77 \mathrm{~K}$ & 6.26 \\
\hline 29 & 6145.02 & $3 p^{3}{ }^{3} \mathrm{D}_{2}^{\mathrm{o}}-5 \mathrm{f}^{3} \mathrm{G}_{3}$ & 5.616 & -29.869 & $-0.82 \mathrm{~K}$ & 6.12 \\
\hline 29 & 6155.14 & $3 p^{3}{ }^{3} \mathrm{D}_{3}^{\mathrm{o}}-5 \mathrm{f}^{3} \mathrm{G}_{4}^{3}$ & 5.619 & -29.869 & $-0.40 \mathrm{~K}$ & 6.73 \\
\hline 30 & 6125.02 & $3 p^{3}{ }^{3} \mathrm{D}_{1}^{\mathrm{o}}-5 \mathrm{f}^{3} \mathrm{D}_{2}^{4}$ & 5.614 & -29.869 & $-0.93 \mathrm{~K}$ & 5.98 \\
\hline 30 & 6142.49 & $3 p^{3}{ }^{3} \mathrm{D}_{3}^{\mathrm{o}}-5 \mathrm{f}^{3} \mathrm{D}_{3}$ & 5.619 & -29.869 & $-0.92 \mathrm{~K}$ & 6.03 \\
\hline 31 & 10843.87 & $4 p^{1} P_{1}^{3}-4 d^{1} D_{2}^{o}$ & 5.863 & -30.145 & $0.15 \mathrm{~N}$ & 7.42 \\
\hline 32 & 10627.66 & $4 p^{1} P_{1}^{1}-4 d^{3} P_{2}^{0}$ & 5.863 & -30.692 & $0.00 \mathrm{~K}$ & 7.11 \\
\hline 36 & 7680.27 & $4 p^{1} P_{1}-5 d^{1} D_{2}^{o}$ & 5.863 & -29.656 & $-0.69 \mathrm{G}$ & 6.94 \\
\hline 43 & 8752.01 & $3 d^{1} D_{2}^{1}-4 f^{1} F_{3}^{2}$ & 5.871 & -30.751 & $-0.52 \mathrm{~K}$ & 7.19 \\
\hline 44 & 8742.46 & $3 d^{1} D_{2}^{o}-4 f^{3} F_{3}^{3}$ & 5.871 & -30.916 & $-0.63 \mathrm{~K}$ & 7.10 \\
\hline 50 & 7034.91 & $3 d^{1} D_{2}^{o}-5 f^{3} G_{3}^{3}$ & 5.871 & -29.027 & $-0.88 \mathrm{G}$ & 6.63 \\
\hline 53 & 10689.73 & $4 p^{3} D_{1}^{2}-4 d^{3} F_{2}^{3}$ & 5.954 & -29.964 & $0.01 \mathrm{~N}$ & 7.42 \\
\hline 53 & 10694.27 & $4 p^{3} D_{2}-4 d^{3} F_{3}^{2}$ & 5.964 & -29.944 & $0.16 \mathrm{~N}$ & 7.56 \\
\hline 53 & 10727.43 & $4 p^{3} D_{3}-4 d^{3} F_{4}^{3}$ & 5.984 & -29.907 & $0.27 \mathrm{~N}$ & 7.75 \\
\hline 53 & 10784.57 & $4 p^{3} D_{2}^{3}-4 d^{3} F_{2}^{0}$ & 5.964 & -29.965 & $-0.72 \mathrm{~N}$ & 6.81 \\
\hline 53 & 10882.83 & $4 p^{3} D_{3}^{2}-4 d^{3} F_{3}^{2}$ & 5.984 & -29.945 & $-0.73 \mathrm{~N}$ & 6.84 \\
\hline 57 & 7918.38 & $4 p^{3} D_{1}^{3}-5 d^{3} F_{2}^{3}$ & 5.954 & -29.663 & $-0.61 \mathrm{G}$ & 6.98 \\
\hline 57 & 7932.35 & $4 p^{3} D_{2}-5 d^{3} F_{3}^{2}$ & 5.964 & -29.663 & $-0.47 \mathrm{G}$ & 7.15 \\
\hline 57 & 7944.00 & $4 p^{3} D_{3}-5 d^{3} F_{4}^{3}$ & 5.984 & -29.663 & $-0.31 \mathrm{G}$ & 7.30 \\
\hline 57 & 7970.30 & $4 p^{3} D_{2}^{3}-5 d^{3} F_{2}^{0}$ & 5.964 & -29.663 & $-1.47 \mathrm{G}$ & 6.19 \\
\hline
\end{tabular}


J. R. Shi et al.: Statistical equilibrium of silicon in the solar atmosphere, Online Material $p 2$

Table 1. continued.

\begin{tabular}{lcccccc}
\hline \hline Mult & $\begin{array}{c}\lambda \\
{[\AA]}\end{array}$ & Line & $\begin{array}{c}E_{\text {low }} \\
{[\mathrm{eV}]}\end{array}$ & $\log C_{6}$ & $\log g f$ & $\begin{array}{c}\log \\
g f \varepsilon_{\odot}\end{array}$ \\
\hline 60 & 6976.51 & $4 \mathrm{p}^{3} \mathrm{D}_{1}-6 \mathrm{~d}^{3} \mathrm{~F}_{2}^{\mathrm{o}}$ & 5.954 & -29.895 & $-1.17 \mathrm{G}$ & 6.50 \\
60 & 7003.57 & $4 \mathrm{p}^{3} \mathrm{D}_{2}-6 \mathrm{~d}^{3} \mathrm{~F}_{3}^{\mathrm{o}}$ & 5.964 & -29.434 & $-0.91 \mathrm{~N}$ & 6.74 \\
60 & 7005.89 & $4 \mathrm{p}^{3} \mathrm{D}_{3}-6 \mathrm{~d}^{3} \mathrm{~F}_{4}^{\mathrm{o}}$ & 5.984 & -29.090 & $-0.75 \mathrm{~N}$ & 6.90 \\
60.02 & 6741.63 & $4 \mathrm{p}^{3} \mathrm{D}_{3}-8 \mathrm{~s}^{3} \mathrm{P}_{2}^{\mathrm{o}}$ & 5.984 & -29.853 & $-1.75 \mathrm{G}$ & 5.94 \\
79 & 8728.01 & $3 \mathrm{~d}^{3} \mathrm{~F}_{2}^{\mathrm{o}}-5 \mathrm{f}^{3} \mathrm{~F}_{3}$ & 6.191 & -29.241 & $-0.61 \mathrm{~K}$ & 7.12 \\
80 & 8595.97 & $3 \mathrm{~d}^{3} \mathrm{~F}_{3}^{\mathrm{o}}-5 \mathrm{f}^{3} \mathrm{G}_{4}$ & 6.181 & -29.125 & $-1.04 \mathrm{~K}$ & 6.64 \\
81 & 7800.00 & $3 \mathrm{~d}^{3} \mathrm{~F}_{2}^{\mathrm{o}}-6 \mathrm{f}^{3} \mathrm{~F}_{2}$ & 6.191 & -29.125 & $-1.29 \mathrm{~K}$ & 6.81 \\
81 & 7849.97 & $3 \mathrm{~d}^{3} \mathrm{~F}_{3}^{\mathrm{o}}-6 \mathrm{f}^{3} \mathrm{~F}_{2}$ & 6.208 & -29.125 & $-0.96 \mathrm{~K}$ & 6.79 \\
\hline 2 & 6347.10 & $4 \mathrm{~s}^{2} \mathrm{~S}_{1 / 2}-4 \mathrm{p}^{2} \mathrm{P}_{3 / 2}^{\mathrm{o}}$ & 8.121 & -30.200 & $0.15 \mathrm{~N}$ & 7.77 \\
2 & 6371.36 & $4 \mathrm{~s}^{2} \mathrm{~S}_{1 / 2}-4 \mathrm{p}^{2} \mathrm{P}_{1 / 2}^{\mathrm{o}}$ & 8.121 & -30.200 & $-0.08 \mathrm{~N}$ & 7.45 \\
\hline
\end{tabular}

